\title{
SOBRE LA REFORMA TRIBUTARIA ESTRUCTURAL QUE SE REQUIERE EN COLOMBIA Reflexiones y propuestas
}

Jorge Espitia a, César Ferrari ${ }^{\mathrm{b}}$, Gonzalo Hernández Isidro Hernández ${ }^{\mathrm{d}}$, Jorge Iván González, Luis Carlos Reyes ${ }^{\mathrm{f}}$ Jairo Orlando Villabona $a^{\mathrm{g}}$ y Gustavo Zafra ${ }^{\mathrm{h}}$

$\mathrm{L}$ a universidad es un lugar donde se crea y difunde el conocimiento a partir de lo ya conocido y de las novedades que puede aportar. No es el único lugar, pero es sin duda uno de los principales. Y ese conocimiento debe contribuir al bienestar de la sociedad y de sus integrantes. Por ello, la universidad no debe estar al margen de los problemas nacionales y de la búsqueda de soluciones.

Uno de esos problemas urgentes es la situación tributaria colombiana. Para enfrentarlo, el gobierno creó una Comisión de Expertos que le presentó una propuesta de reforma a comienzos de 2016. A partir de ella elaboró su propia propuesta y presentó un proyecto de ley al Congreso en octubre 2016, el cual aprobó la reforma tributaria mediante la Ley 1819 en diciembre de 2016.

a Profesor de la Escuela Colombiana de Ingeniería, Bogotá, Colombia, [jeespitia@gmail.com].

b Profesor de la Pontificia Universidad Javeriana, Bogotá, Colombia, [ferrari@ javeriana.edu.co].

c Profesor de la Pontificia Universidad Javeriana, Bogotá, Colombia, [gonzalo. hernandez@javeriana.edu.co].

d Profesor de la Universidad Externado de Colombia, [isidro.hernandez@ uexternado.edu.co].

e Profesor de la Universidad Externado de Colombia, [jorgeivangonzalez29@ gmail.com].

f Profesor de la Pontificia Universidad Javeriana, Bogotá, Colombia, [luis_reyes@javeriana.edu.co].

g Profesor de la Universidad Nacional de Colombia, [jovillabonar@unal.edu.co].

h Profesor de la Pontificia Universidad Javeriana, Bogotá, Colombia, [gzafra@ javeriana.edu.co]. Fecha de recepción: 20-11-2016, fecha de modificación: 2302-2017, fecha de aceptación: 15-04-2017. Sugerencia de citación: Comisión Académica de Reforma Tributaria Estructural, "Sobre la reforma tributaria estructural que requiere Colombia”, Revista de Economía Institucional 19, 36, 2017, pp. 149-174. DoI: https://doi.org/10.18601/01245996.v19n36.06. 
Preocupados por la difícil situación nacional y sin otra razón que contribuir desinteresadamente a mejorar las decisiones de política económica para lograr el bienestar de los colombianos, ocho profesores interesados en temas tributarios, fiscales y de política económica de cuatro universidades con sede en Bogotá nos constituimos en Comisión Académica e hicimos algunas reflexiones y propuestas sobre la reforma tributaria estructural que a nuestro entender el país requiere. Este escrito presenta esas reflexiones y propuestas, y algunos comentarios sobre la reforma aprobada.

\section{EL CONTEXTO Y LAS PREGUNTAS QUE SE DEBEN RESPONDER}

En un contexto macroeconómico global muy complejo, en especial para economías en desarrollo como la colombiana debido a la caída de los precios internacionales de materias primas, que reduce el ingreso de divisas, devalúa la tasa de cambio e incrementa los precios de los bienes y servicios transables, la economía se desacelera y los ingresos tributarios disminuyen. Se estima que en 2016 esa reducción se tradujo en Colombia en un déficit fiscal de unos 20 billones de pesos. Es entonces conveniente revisar la situación tributaria del país.

Es claro que para evitar de manera permanente las dificultades mencionadas se debe modificar la estructura productiva para hacerla menos dependiente de las materias primas y de sus inestables precios internacionales. Desde esta perspectiva, es pertinente impulsar la industria, la agricultura y el ingreso de turistas. Es también clara la conveniencia de flexibilizar la regla fiscal estricta en un entorno donde los precios del petróleo caen, el ingreso disminuye y la economía se desacelera, pues mantenerla sería una medida procíclica que profundizaría los efectos del ciclo económico. Por supuesto, esos cambios van más allá de una reforma tributaria estructural.

En la coyuntura política, económica y social colombiana, el tema tributario es sumamente importante. Parece haber gran consenso en la necesidad de una reforma tributaria que aumente el recaudo fiscal para cerrar la brecha histórica entre el gasto y los ingresos tributarios, proveer a los ciudadanos los bienes públicos esenciales (vivienda, agua potable, salud, educación, entre otros), of recer al sector privado los bienes públicos para que la economía sea más competitiva, cumplir a las víctimas del conflicto armado los compromisos adquiridos y mejorar la distribución del ingreso.

No sobra señalar que la política fiscal de los últimos gobiernos ha sido más procíclica que contracíclica. En general, se espera que en 
una época de bonanza económica se avance en la política tributaria y que el gasto público aumente cuando la economía se desacelera. En otras palabras, la política fiscal debería reducir el ingreso disponible cuando la economía está en auge y aumentarlo para enfrentar una crisis o una desaceleración.

Cuando la economía se desacelera por las razones indicadas, la propuesta de reforma que parece tener más aceptación política es aumentar la tributación a los ciudadanos, en particular a los asalariados, por dos vías: un mayor impuesto de renta y un mayor impuesto al consumo de bienes y servicios (aumentando las tarifas del impuesto al valor agregado (IVA) y del impuesto al consumo). Estas dos medidas no son convenientes porque profundizarían la reducción del ingreso disponible de las familias y la concentración del ingreso.

No obstante, la reforma tributaria estructural es urgente y debe responder las preguntas usuales en el análisis de la tributación óptima: suficiencia, eficiencia, progresividad y simplicidad. Tales preguntas son:

1. ¿Cuál debería ser la principal fuente de recaudo?

2. ¿Qué impuestos deberían considerarse y cuáles descartarse?

3. ¿Con qué criterios y a qué tasas deberían fijarse los impuestos?

4. ¿Cuán simple/compleja debe ser la estructura tributaria?

5. ¿Cuál debería ser la meta de recaudo como proporción del PIB?

6. ¿Cuál sería la estructura institucional que asegure ese recaudo?

7. ¿Cuáles deberían ser las principales medidas para asegurarlo?

\section{SOBRE LAS CARACTERÍSTICAS DEL SISTEMA ACTUAL}

Las respuestas a estas preguntas deben partir, necesariamente, de reconocer la situación tributaria, que pese a la Reforma de 2016 no parece haber cambiado mucho:

- La tributación es insuficiente para proporcionar los bienes públicos esenciales que demandan los ciudadanos y para construir la infraestructura económica que el país demanda con urgencia.

- E1 recaudo es aún más insuficiente en un momento en que se inicia la construcción de una paz duradera con la implementación del Acuerdo de Paz entre el gobierno colombiano y las farc. El conflicto armado, que ha durado más de cinco décadas, ha provocado 220 mil muertos, 6,8 millones de desplazados (el 14\% de la población total), inconmensurables dolores y temores, y grandes deudas con las víctimas (viviendas, indemnizaciones, etc.), que la 
Corte Constitucional ha declarado población vulnerable, mientras que está desfinanciada la Ley 1448 de $2011^{1}$.

- Las cifras de recaudo no dejan dudas. Según la Organización para la Cooperación y el Desarrollo (OCDE), mientras que en 2014 la tasa de recaudo en Colombia era del 20,3\% del PIB, la tasa latinoamericana promedio era del $21,7 \%$ y la de los países de la ocDE del 34,4\%. Colombia aspira a pertenecer a esta organización internacional.

- La tributación es ineficiente. En general, las tasas nominales para las empresas no son competitivas a nivel internacional pues superan notoriamente las que pagan en países similares o donde se domicilian los principales competidores de los productores colombianos de bienes y servicios. En 2016 la tasa nominal del impuesto de renta de las empresas, combinada y con diversos nombres, era de un $40 \%$ y habría llegado al $43 \%$ en 2018 . Cabe señalar que la Reforma de 2016 redujo al 34\% la tasa nominal del impuesto de renta de las empresas y eliminó el impuesto sobre la renta para la equidad (CREE).

- No obstante dichas tasas nominales, las tasas que muchas empresas pagaban efectivamente eran bastante menores, con sus consiguientes efectos regresivos y negativos sobre el recaudo. La sobrecarga tributaria nominal a las empresas induce a que muchas busquen todo tipo de excepciones y prebendas para reducir la tasa nominal a una tasa efectiva mucho menor o a que simplemente evadan el pago cargando a la empresa gastos ajenos para reducir su base gravable. Así, mantienen contabilidades poco transparentes que les impiden recurrir al mercado de capitales para financiarse.

- Gran parte del recaudo tributario sigue basado en tasas e impuestos anti técnicos, como el gravamen a los movimientos financieros.

- La tributación es inequitativa. Las personas naturales que poseen más riquezas e ingresos no son las que proporcionalmente más contribuyen al recaudo fiscal. Más aún, las personas naturales casi no contribuyen, casi toda la contribución tributaria recae sobre personas jurídicas, al contrario del mundo desarrollado. En los países de la OCDE por cada dólar recaudado a personas jurídicas se recaudan 2,3 dólares a personas naturales; en Colombia se recaudan apenas 0,22 .

- La deficiente estructura del sistema con respecto a las personas naturales se manifiesta en una elevada carga tributaria cuando sus

\footnotetext{
1 "Por la cual se dictan medidas de atención, asistencia y reparación integral a las víctimas del conflicto armado interno y se dictan otras disposiciones".
} 
ingresos principales provienen de remuneración al trabajo, y una poca o nula tributación cuando provienen de rentas de capital y dividendos. Cabe señalar que la Reforma de 2016 reintrodujo el impuesto a los dividendos, con una tasa del 5\% a partir de 19,1 millones de pesos y del 10\% a partir de 31,8 millones (para 2017). Estos valores se incrementarán anualmente con el ajuste de la unidad de valor tributario (UVT), hoy en 31.859 pesos.

- Así, la estructura tributaria no mejora la distribución del ingreso, sumamente concentrada en Colombia y una de las más desiguales del mundo. Otros países la modifican en forma sustantiva mediante la progresividad, de los impuestos y de los subsidios, y la mayor contribución de las personas naturales.

- Además, la tributación recae sobre unos pocos. La evasión es un problema extendido y de suma importancia en el país. Aunque las empresas del sector formal enfrentan una carga tributaria nominal elevada y compleja, solo una pequeña parte de las personas jurídicas y naturales paga impuestos de renta y patrimonio.

\section{SOBRE LA ESTRUCTURA TRIBUTARIA DESEABLE}

- E1 recaudo tributario debe satisfacer las crecientes necesidades de gasto público. En el largo plazo, el objetivo de suficiencia debe ser que las principales responsabilidades del Estado -seguridad externa e interna, justicia, gasto social, es decir educación y salud, e infraestructura- sean cumplidas en forma satisfactoria.

- La estructura tributaria debe ser simple, clara y debidamente divulgada. No obstante, esa simplicidad no debe obviar cierta complejidad cuando los requerimientos de equidad la demanden. La simplicidad extrema lleva a una unificación de tasas que contradice la progresividad. En otras palabras, no se trata de establecer una sola tasa de IVA, de impuesto a las utilidades o de renta, sino varias, sin que sean numerosas, para hacer progresiva la estructura tributaria. Un sistema tributario excesivamente simple no puede ser equitativo. Por ello, en la definición de las normas tributarias es necesaria cierta complejidad que, no obstante, debe ser clara y transparente, es decir, con un Estatuto Tributario breve y sencillo.

- Además, debe compensar el agotamiento de algunas fuentes de ingreso fiscal debido a choques exógenos, como la caída de los precios internacionales del petróleo y del carbón, que ha disminuido el recaudo del impuesto de renta de las empresas y las regalías que financian gran parte de la inversiones de los departamentos y municipios. 
- La estructura tributaria debe centrarse en los impuestos directos. La actual está sesgada hacia los impuestos indirectos, en particular hacia el IVA, de modo que es altamente regresiva.

- El recaudo debería recaer en forma progresiva sobre la renta y la riqueza de personas naturales más que sobre las de las empresas, como en los países desarrollados, en particular sobre ganancias de capital y dividendos, y en menor grado sobre la remuneración al trabajo.

- Los impuestos no deberían contemplar exenciones fiscales para empresas y personas naturales pues son inequitativos. La alta concentración de esos beneficios en pocas empresas contribuye a concentrar la riqueza y el ingreso. Así, no tiene sentido reducir la tarifa para las empresas sin gravar los dividendos que reciben los dueños del capital y eliminar las exenciones fiscales que corroen la base gravable del impuesto de renta de las personas jurídicas.

- Los impuestos al suelo y al urbanismo tienen un enorme potencial que no se ha aprovechado. Es necesario actualizar los catastros y crear incentivos para que las ciudades grandes y medianas mejoren los recursos provenientes de la dinámica urbana (participación en plusvalías, valorización, cargas, etc.). Sin restar autonomía a los municipios, la reforma tributaria puede incluir disposiciones que favorezcan el aumento de los tributos locales. Este es un componente fundamental de la integralidad del sistema tributario.

\section{SOBRE LA META DE RECAUDO Y EL GASTO FISCAL}

- La meta de recaudo debe ser la necesaria para financiar el creciente gasto fiscal derivado de las necesidades sociales insatisfechas durante décadas, siempre y cuando las tasas sean competitivas, es decir comparables a nivel internacional. Sería deseable que en 2020 el recaudo alcance al menos el 25\% del PIB, aun menor que la tasa media $(30,9 \%)$ de los países que la OCDE incluye en sus estadísticas.

- Una de las razones para aumentar los impuestos es la necesidad de financiar bienes y servicios públicos que tienen costos crecientes. A medida que las sociedades se hacen más complejas, la participación del Estado tiende a aumentar y los costos de esa intervención son marginalmente crecientes. Esta tendencia es clara en educación, ciencia y tecnología, salud, seguridad, medio ambiente y regulación.

- En educación, la conjunción de mejor calidad y mayor cobertura solo se logra incurriendo en costos marginales crecientes. Si Co- 
lombia aspira a ser el país más educado de América, debe aumentar el gasto por niño al menos a un nivel similar al de Chile, según la OCDE de 4.551 dólares en primaria y 4.495 dólares en secundaria en 2011, que a la tasa de cambio actual representan unos 12 millones de pesos por niño año, apenas la mitad del promedio de los países de la ocDE. Colombia gasta 2,5 millones de pesos por niño año (Bogotá 4 millones). La brecha con respecto a Chile es notoria y con respecto a la OCDE abismal.

- Los costos marginales crecientes son más notorios en ciencia, tecnología e innovación. E1 atraso de Colombia es significativo y una manera importante de superarlo es recurrir al gasto público. La investigación de punta implica costos crecientes que las empresas privadas usualmente no asumen. Además, así no haya investigación de primera línea, el manejo y la adaptación de las tecnologías existentes requiere gastos marginalmente crecientes. Los niveles de gasto varían dependiendo del país, las instituciones, etc.

- En salud, el aumento de la esperanza de vida también es más costoso en el margen. El aumento de 30 a 35 años fue posible por la construcción de alcantarillados, la aplicación masiva de vacunas, la extensión de la cobertura de agua potable, etc. Estas inversiones son relativamente menores a las que hoy se requieren para aumentarla en un solo año, digamos de 77 a 78 años. Y la financiación de los costos de la salud, cuando las intervenciones son complejas, tiene que ser garantizada por el Estado, así que el gasto público debe aumentar.

- En seguridad los costos también están aumentando porque la vigilancia requiere tecnologías cada vez más complejas.

- En medio ambiente se siguen posponiendo decisiones que en el mediano plazo serán mucho más costosas. Los bosques se están perdiendo, los ríos se siguen contaminando, los humedales se rellenan, los páramos se invaden, etc. Tarde o temprano habrá que hacer frente a estos daños ambientales, y el costo de la intervención tiene que ser financiado con gasto público.

- La regulación adquiere mayores niveles de complejidad y el Estado necesita una burocracia mejor preparada con instrumentos de supervisión adecuados. Estos procesos se tienen que financiar con impuestos.

- En síntesis, ante los costos crecientes no hay otra opción que recurrir a los impuestos. La riqueza de la sociedad es la fuente última de financiación pública. 


\section{SOBRE LA REFORMA TRIBUTARIA ESTRUCTURAL}

- De lo anterior se deduce la necesidad de una reforma tributaria estructural que conduzca a un nuevo Estatuto Tributario, coherente con el Estado Social de Derecho y los lineamientos de descentralización estipulados en la Constitución de 1991. La reforma debe llevar a un cambio profundo en la orientación y las preferencias del actual sistema tributario.

- En ese sentido, no se deben hacer más reformas tributarias parciales, que han sido numerosas, casi una por año en las últimas décadas, y en su mayor parte inoficiosas.

- Se debe entender que la reforma estructural es un acto político, que sus normas afectan intereses no siempre compatibles, y que es necesario superar los intereses particulares que conspiran contra una legislación tributaria suficiente, eficiente, progresiva y simple. Para ello se debe convocar y convencer a las fuerza políticas representadas en el Congreso.

\section{SOBRE EL IMPUESTO A LAS UTILIDADES DE LAS EMPRESAS} Y A LA RENTA DE LA PERSONAS NATURALES

- Cuando las personas naturales se clasifican de acuerdo con su nivel de ingresos, según la Dirección de Aduanas e Impuestos Nacionales (DIAN), en 2015 unos dos millones de declarantes del impuesto de renta (2.022.014) tenían un ingreso anual inferior a mil millones de pesos, 23.043 un ingreso entre mil millones y diez mil millones y 628 un ingreso superior a diez mil millones.

- Cuando se calculan las tarifas nominales medias y el peso de los impuestos en el total de ingresos con información de la DIAN, se encuentra que en 2015 las tarifas nominales oscilaban entre el $8,3 \%$ y el $31,3 \%$, y el peso de los impuestos entre el $2,8 \%$ y el $1,4 \%$ para personas naturales con ingresos de más de 10 mil millones de pesos. De modo que la tarifa efectiva es mucho menor que la tarifa nominal. Para las personas naturales de menores ingresos, las deducciones se concentran en vivienda y pensiones.

- Considerando el ingreso mensual de las personas naturales que declararon renta ante la DIAN entre 2009 y 2014 se deduce que: 1) la concentración del patrimonio y del ingreso ha aumentado en forma notable; las personas naturales más ricas son cada vez más ricas; 2) la relación entre patrimonio e ingresos ha aumentado en los deciles más ricos; 3 ) si se compara el peso del valor del impuesto pagado con respecto al nivel de ingreso se observan grandes in- 
equidades verticales: mientras que en el decil 1 el impuesto pagado representa el 2,7\%, en los deciles 4 y 5 representa el 1,5\%.

- El gobierno colombiano ha hecho esfuerzos para incluir cada vez más contribuyentes. Según información de la DIAN, en 2009 el ingreso medio gravable del decil más bajo era de 6.660 .816 pesos mensuales. De acuerdo con las últimas declaraciones de renta, en 2014 era de 3.268 .750 pesos mensuales. En otras palabras, entre 2009 y 2014 la base mínima gravable se redujo de manera considerable. Esta tendencia no parece conveniente porque no favorece la equidad. En vez de aumentar la tarifa a los niveles de ingresos superiores se amplía la base en los ingresos inferiores.

- No conviene reducir ese nivel de ingreso tal como se ha propuesto. Pues muy seguramente ampliaría notablemente la base de contribuyentes y, por tanto, los costos de administración y fiscalización en que incurre la DiAn. Además, esa medida no favorece la equidad. Es muy probable que los costos de administración y fiscalización no guarden relación con el recaudo que se obtendría de los nuevos contribuyentes.

- La reducción de la base mínima gravable que se estableció en los años anteriores no cambió la composición del recaudo entre personas naturales y personas jurídicas. Se puede augurar que hoy tampoco lograría ese cambio: en la distribución del recaudo entre personas naturales y personas jurídicas por impuestos como el de renta, la relación era de 1 a 3 y en patrimonio de 1 a 10 en 2015 . Cerca del $80 \%$ de estos dos impuestos se concentraba en personas jurídicas.

- Si la tarifa media del impuesto de renta de cada decil se incrementara en 5 puntos porcentuales, el recaudo adicional que se obtendría entre personas naturales sería de unos tres billones de pesos. Mucho menos de lo que se obtendría si se grava el $25 \%$ de las exenciones fiscales a las personas jurídicas, que ascienden a unos 8,5 billones de pesos.

- Por otro parte, pensar que el aumento de las tarifas para personas naturales cambiaría sustancialmente la participación entre personas jurídicas y naturales es también erróneo. $\mathrm{El}$ recaudo sería mayor si se eliminan las exenciones fiscales a las personas jurídicas que si se aumentan sustancialmente las tarifas a los ciudadanos.

- Lo que se requiere es mejorar la equidad vertical entre personas naturales. Como muestran las tarifas medias (recaudo/base gravable) y la relación entre recaudo e ingresos, esa inequidad es profunda.

En consecuencia: 
- Debería superarse el impuesto de renta al que están obligadas las personas naturales y jurídicas que tienen muchas exenciones, y eliminarse sus sustitutos parciales con menores exenciones, como el impuesto mínimo alternativo (IMAN), el impuesto mínimo alternativo simple (IMAS), el impuesto sobre la renta para la equidad (CREE) y su sobretasa. En opinión nuestra, se deberían eliminar todos ellos y establecer en su lugar un impuesto a las utilidades de las personas jurídicas y otro impuesto a los ingresos netos de las personas naturales, sin exenciones, porque son personas distintas y sus ingresos tienen orígenes distintos. Cabe señalar que la Reforma de 2016 eliminó el IMAN, el imas, el CREE y su sobretasa.

- Por simplicidad y porque deben ser competitivas a nivel internacional, las tasas del impuesto a las utilidades de las personas jurídicas deberían ser dos: del 25\% para las empresas grandes y del 15\% para las pequeñas y medianas, sin exenciones.

- Las tasas del impuesto a los ingresos netos de las personas naturales deben ser progresivas a partir de cierto nivel, el cual cubriría los gastos necesarios del contribuyente, y llegar a tasas máximas del $50 \%$. Los ingresos deben incluir los de todas las fuentes, incluidos los dividendos repartidos por las empresas. Las tasas para personas naturales deben ser mayores que para las empresas a fin de incentivar una menor distribución de dividendos e inducir mayores tasas de inversión.

Cuadro 1

Impuesto a la renta de personas naturales

\begin{tabular}{llc}
\hline UVT & Pesos & Tasa marginal (\%) \\
\hline De 0 a 1.345 & De 0 a 40.017.785 & 0 \\
\hline$>1.345$ a 2.353 & $>40.017 .785$ a 70.008 .809 & 10 \\
$>2.353$ a 3.362 & $>70.008 .809$ a 100.029 .586 & 20 \\
$>3.362$ a 4.370 & $>100.029 .586$ a 130.020 .610 & 30 \\
$>4.370$ a 5.000 & $>130.020 .610$ a 148.765 .000 & 33 \\
$>5.000$ a 6.386 & $>148.765 .000$ a 190.002 .658 & 35 \\
$>6.386$ a 10.000 & $>190.002 .658$ a 297.530 .000 & 40 \\
$>10.000$ a 30.000 & $>297.530 .000$ a 892.590 .000 & 45 \\
$>30.000$ & $>892.590 .000$ & 50 \\
\hline
\end{tabular}

UVT $=29.753$ pesos (enero de 2016).

- La construcción del cuadro 1, del impuesto a los ingresos netos de las personas naturales, considera los siguientes criterios adicionales: 1) no se debe empeorar el bienestar de los receptores de ingresos por trabajo; 2) los ingresos laborales iguales o mayores a 10 salarios mínimos legales vigentes (SMLV) pueden ser equivalentes al ingreso de una persona natural que obtiene su ingreso de capital en negocios con ventas mensuales de 30 millones de pesos, por 
ejemplo, de una carnicería en Bogotá; 3) para no afectar el consumo básico, una parte de la población debe quedar exenta, lo que implica incluir un mínimo exento; 4) para asegurar la equidad se deben gravar los ingresos de cualquier fuente, incluidos los dividendos distribuidos por las empresas.

- La idea central de nuestra propuesta es eliminar todo tipo de exenciones fiscales y deducciones. El recaudo que se lograría con esa eliminación sería una gran fuente de recursos para proporcionar a la sociedad colombiana los bienes públicos esenciales para mejorar su calidad de vida y atender las necesidades de la población más vulnerable. Si por razones justificadas se hiciesen algunas excepciones tributarias sería preferible reconocerlas como subsidios e incorporarlas expresamente en el gasto público.

\section{SOBRE EL IMPUESTO AL PATRIMONIO Y A LAS GANANCIAS OCASIONALES}

- El impuesto al patrimonio es conveniente por una razón elemental: fomentar su uso productivo. Si no se usa y, por tanto, no produce ingreso, tarde o temprano el impuesto acaba siendo mayor que su valor nominal. El patrimonio debe incluir los tangibles e intangibles a su valor comercial, incluidos los activos financieros.

- El impuesto al patrimonio debería aplicarse a las personas naturales y no a las empresas. El impuesto progresivo al patrimonio de las personas naturales es efectivo para mejorar la equidad en la distribución del ingreso. Aplicarlo a las empresas es contraproducente, pues al reducir su rentabilidad reduce sus recursos de inversión y, por tanto, el crecimiento de la economía.

- El impuesto al patrimonio debe recaer sobre su valor neto (descontado el saldo de crédito vigente para adquirirlo), a tasas progresivas por encima de cierto valor, y ser concordante con los impuestos prediales, al suelo, al urbanismo y a los automóviles, que se deben considerar como un adelanto de este impuesto. Para definir el patrimonio gravable parece sensato seguir excluyendo del total la casa de habitación, hasta por un valor de 400 millones de pesos, unas 13.445 uvT en 2016.

- Para definir mejor las tasas adecuadas de impuesto al patrimonio conviene revisar la experiencia internacional:

- En España el impuesto se cobra a partir de 700 mil euros, con una exención por patrimonio inmobiliario de 300 mil euros, y con tasas entre el $0,2 \%$ y el $2,5 \%$. 
- En Francia se cobra mediante el Impuesto de Solidaridad sobre la Fortuna a partir de 1,3 millones de euros, con tasas progresivas entre el $0,5 \%$ y el $1,5 \%$.

- En Noruega las tasas son progresivas, entre el 0,9\% y el 1,1\%.

- En Suiza la tarifa progresiva es del 0,81\% al 3,08\%.

- En Argentina se cobra con tasas del 0,5\% al 1,25\%.

- En Colombia sus límites inferior y superior se podrían definir a partir del promedio simple de las tarifas de los países de la muestra. Así, el límite inferior podría ser del 0,5\% y el superior del 2,0\%. El cuadro 2 muestra la posible estructura de estas tasas.

- El impuesto a las ganancias ocasionales por compra y venta de activos, incluidos los activos financieros, por su valorización, y por herencias deberían ser fundamentales a partir de cierto nivel. Las primeras, salvo cuando provengan de la venta de la vivienda única o del primer automóvil que se deben exceptuar, deben tener tasas del orden del $25 \%$. Las herencias superiores a cierto nivel deberían tener tasas de un $40 \%$.

Cuadro 2

Tasas de impuesto al patrimonio

(Millones de pesos y porcentaje)

\begin{tabular}{lc}
\hline Rango & Tasa marginal \\
\hline De 1.100 a 2.000 & $0,5 \%$ \\
$>2.000$ a 3.000 & $1,0 \%$ \\
$>3.000$ a 6.000 & $1,5 \%$ \\
$>6.000$ & $2,0 \%$ \\
\hline
\end{tabular}

\section{SOBRE EL IMPUESTO AL VALOR AGREGADO Y AL CONSUMO}

- Con la reforma de diciembre de 2016, la tasa general del iva aumentó del 16\% al 19\% para la mayor parte de los bienes y servicios.

- No era sensato ese aumento que se sugirió y finalmente se aprobó. Más bien convendría reducirla, por equidad, porque gran parte ya estaban exceptuados y pronto será imposible controlar las operaciones virtuales e informales en casi todos los sectores de la economía. Pero, dadas las actuales dificultades fiscales, esa reducción debería ser progresiva y postergarse hasta que se implementen en forma adecuada los impuestos de renta y patrimonio antes mencionados. Solo se debería haber aumentado por razones de salud pública, incluso a más del 19\%, para reducir el consumo de bebidas azucaradas y tabaco.

- Aunque parece necesario aumentar el recaudo, no se debe aumentar a costa de la equidad, más aún dada la actual inequidad en la 
distribución del ingreso. En cambio, se deberían eliminar todas las exenciones al IVA -en las que con frecuencia se refugian intereses especiales-, salvo a la canasta familiar: alimentos, medicinas, educación, vivienda, agua y electricidad. A esa canasta se deberían añadir los servicios de telefonía fija y móvil y de Internet fijo y móvil, de uso masivo: para desempeñarse de modo eficiente en el mundo moderno hoy se requieren comunicación e información.

- Aumentar el iva al 19\% ha convertido a Colombia en uno de los países en desarrollo con una de las tasas más altas. Es erróneo pensar que un IVA de nivel europeo es un signo de progreso. Por el contrario, agravará la desigualdad, que en Europa no alcanza iguales proporciones y es contrarrestada por la existencia de Estados de Bienestar muy sólidos. Mientras Colombia no tenga un Estado de Bienestar semejante, que proporcione a todo ciudadano educación y salud de alta calidad, y una red de seguridad social que proteja de manera efectiva a los desempleados y a las personas vulnerables, es perjudicial aumentar la desigualdad imponiendo un IVA similar al europeo. Para crear ese Estado de Bienestar, el país debería apoyarse en impuestos directos y progresivos, como los impuestos de renta y patrimonio ya mencionados.

- El iva es ciertamente un impuesto regresivo; en la práctica grava con un porcentaje más alto a los pobres que a los ricos. Tratándose de un impuesto al consumo y dado que, en general, los pobres no ahorran, pagan por IVA un porcentaje de sus ingresos más alto que el que pagan los ricos a quienes, aun después de satisfacer sus necesidades básicas, sí les quedan recursos para ahorrar.

- Para disminuir esa regresividad es conveniente fortalecer dos de las soluciones que existen en Colombia y en el mundo: la exención de la canasta familiar y un umbral por debajo del cual los negocios no deben pagar el IVA. Cuando se exime la canasta familiar, se reduce la carga impositiva de los pobres, que gastan casi todo su ingreso en bienes y servicios de esa canasta. A su vez, la reducción del impuesto es menor para los ricos, que consumen más bienes de otro tipo, incluidos los de lujo. También se debería eximir del IVA a los pequeños negocios. El umbral por debajo del cual están exentos podría triplicarse, sin que por ello deje de estar alineado con las prácticas internacionales.

\section{SOBRE EL GRAVAMEN FINANCIERO}

- El gravamen a los movimientos financieros (GMF), hoy del 4 por mil, hace una contribución importante al recaudo como porcen- 
taje del PIB, pero sus costos, en términos de desintermediación financiera y reducción de la competitividad del sistema, superan su eficiencia como fuente de ingresos. Debería desaparecer progresivamente.

\section{SOBRE EL IMPUESTO A LAS REMESAS}

- En Colombia, el impuesto a las remesas se abolió en 2007, lo que puso en desventaja a los inversionistas nacionales frente a los extranjeros. Esa situación induce fuga de capitales: es más ventajoso invertir desde el extranjero porque el inversionista del exterior no paga impuestos cuando envía las ganancias fuera del país, y no son patrimonio gravable, como sí lo son para los inversionistas nacionales.

- El impuesto a las remesas existe en casi todos los países. Para definir las tasas adecuadas conviene revisar la experiencia internacional:

- En Chile existe un impuesto equivalente al de remesas, el impuesto adicional. En 2016 las empresas chilenas pagaron una tarifa general del 24\%; este impuesto se denomina de primera categoría. Si son extranjeras, cuando giran dinero al exterior la tarifa es del $35 \%$, a la que se resta la parte proporcional del pago por el impuesto de primera categoría.

- En España el cobro de impuestos a los no residentes que tienen un establecimiento permanente en territorio español tiene un gravamen del $30 \%$ sobre los beneficios del establecimiento (esto es, sobre la diferencia entre sus ingresos y gastos a efectos fiscales), con ciertas especificidades en casos concretos.

- En Estados Unidos, si no hay un tratado tributario con otro país que of rezca beneficios, una compañía extranjera puede estar sujeta no solo a una tasa de impuestos de renta de un $40 \%$ (considerando los impuestos de Florida y de Estados Unidos), sino que, dependiendo de las circunstancias, con ingresos provenientes de inversiones en Estados Unidos, puede estar sujeta a un impuesto federal adicional, el "impuesto sobre utilidades de una sucursal" (Branch Profits Tax). La tasa del 30\% se aplica a los ingresos obtenidos en Estados Unidos que la compañía extranjera no distribuye como dividendo o no reinvierte en activos estadounidenses.

- De modo que no hay razones para que en Colombia no exista este impuesto. Sería sensato que, para los dividendos, cuando se gire el dinero al exterior se aplique una tarifa del 35\%, como en Chile, 
a la que se reste la parte proporcional del impuesto pagado por la sucursal. E1 cuadro 3 ilustra este planteamiento.

Cuadro 3

Impuesto a las remesas, ejemplo ilustrativo

\begin{tabular}{ll}
\hline Utilidades de una compañía extranjera en Colombia & 1.000 \\
Impuesto de renta (25\%) & $(250)$ \\
Distribución de utilidades & 750 \\
Dividendos recibidos por un no residente & 750 \\
Más impuesto de primera categoría agregado a la base & 250 \\
Base del impuesto adicional & 1.000 \\
Impuesto adicional (35\%) & 350 \\
Impuesto de renta pagado por la sucursal & $(250)$ \\
Impuesto a pagar por la remisión & 100 \\
\hline
\end{tabular}

\section{SOBRE LOS IMPUESTOS LOCALES}

- Aunque nuestra propuesta se refiere ante todo a los impuestos de tipo nacional, mencionamos criterios sobre los impuestos locales (municipales y departamentales) porque: 1) es necesario captar el impacto de la combinación de impuestos nacionales y locales sobre el contribuyente; 2) la oferta de bienes y servicios públicos puede ser más eficiente y tener mayor cobertura si hay coordinación tributaria entre instancias nacionales y locales; 3) el gran potencial fiscal del país está en los impuestos al suelo y al urbanismo.

- En cuanto al predial, es necesario actualizar los valores catastrales, relacionar la tarifa con el avalúo y no con el estrato, y aumentar la tarifa en forma progresiva con el valor del inmueble.

- El valor catastral debería seguir al comercial. En condiciones ideales debería ser un 85-90\% del valor comercial del inmueble (como lo es hoy en Bogotá). Es urgente actualizar los catastros y muy conveniente integrar catastros y registros, para lo cual el Instituto Geográfico Agustín Codazzi (IGAC) debe contar con los recursos necesarios para actualizar catastros y modernizar la información geo-referenciada. A todas luces es un excelente negocio invertir en la modernización del IgAc.

- Los municipios cobran diferentes tarifas del predial, con criterios muy heterogéneos: algunos la fijan por estrato, otros combinan estrato y avalúo catastral, otros solo consideran el avalúo catastral. En algunos casos la tarifa es plana, en otros es ligeramente progresiva y va aumentando con la base. Una vez actualizados los catastros, esta debería ser la base única del impuesto y aumentar la tarifa con el valor del inmueble. Como criterio general, sería preferible que el aumento no fuera proporcional sino progresivo. El estrato no debería ser la base para fijar el impuesto. 
- Hay casos escandalosos en lo que respecta a la diferencia entre las tarifas nominal y efectiva del predial. Los datos indican que se puede aumentar el recaudo acercando la tarifa efectiva a la nominal.

- En cuanto al impuesto de industria y comercio (ICA), la situación es compleja en todos los municipios y las tarifas muy dispersas. Es conveniente simplificarlo, reduciendo la dispersión de tarifas, agrupando las actividades gravadas conforme a las diferencias en rentabilidad y definiendo mejor la cobertura territorial. En los municipios las tarifas se diferencian por sector económico, una clasificación inadecuada. Sería mejor determinarlas usando como base gravable el ingreso bruto.

- Las obligaciones urbanísticas, las cargas, la participación en la plusvalía y las contribuciones por valorización ya están incluidas en las normas vigentes. Aunque son una fuente importante de recursos para los municipios no se utilizan de manera adecuada. Se debe incentivar a los municipios para que usen estas fuentes de financiación.

\section{SOBRE LA CONCORDANCIA ENTRE TRIBUTACIÓN NACIONAL Y LOCAL}

- Es necesario simplificar la compleja estructura del sistema tributario nacional y hacer que concuerde con impuestos locales como el ICA, el predial, el de vehículos automotores, etc., para evitar una tributación excesiva y repetitiva de personas naturales y empresas. Los impuestos predial y de automóviles se deberían deducir del impuesto al patrimonio. El ICA solo se debería imponer a las empresas y considerarlo como un gasto a fin de reducir la base gravable del impuesto a las utilidades.

- Las medidas para asegurar una buena estructura fiscal deben considerar la desarticulación existente entre tributación nacional y local, que genera grandes costos de administración para el gobierno nacional y local, así como para las empresas.

- A nivel local se deben eliminar los impuestos que tienen un recaudo mínimo, como las estampillas, debido a su carácter regresivo y a que recaen sobre los contribuyentes más pobres. Se debe, además, revaluar el sistema tributario territorial, dado que existe una amplia variedad de impuestos que recaen en forma inequitativa sobre los contribuyentes, sin que necesariamente se beneficien del servicio, como los de alumbrado público y estampillas.

- En los municipios pequeños el predial debería ser recaudado por una entidad nacional, que lo trasladaría al respectivo municipio. 


\section{SOBRE LA GESTIÓN TRIBUTARIA EN COLOMBIA}

- No puede proponerse una reforma tributaria eficiente y eficaz al margen de propuestas para mejorar la organización responsable del recaudo y fiscalización de la tributación y de las aduanas, la DIAN, cuya gestión fiscal es insatisfactoria: no ha reducido el fraude, la evasión ni la elusión fiscal de manera sustantiva.

- La reestructuración de la DiAn debería prepararla para atender la recomposición de impuestos propuesta. Hoy se orienta a cubrir impuestos indirectos, y usualmente es más complejo recaudar impuestos directos. La reestructuración debe dotarla, como se detalla más adelante, de una base informativa fiscal amplia y suficiente que incluya información fiable de los accionistas de las empresas y de la distribución de utilidades. Aparejada con el requerimiento de una contabilidad transparente de las firmas; no tiene sentido que el "total de deducciones" contables corresponda a "gastos operacionales de administración" y a "gastos operacionales de ventas", que en 2015 sumaron cerca de 480 billones de pesos.

- Una investigación sobre la DIAN arrojó resultados insatisfactorios debidos a falta de personal y capacitación adecuada, falta de cooperación institucional e internacional, de penas por evasión, mala disposición y educación fiscal, así como por la inexistencia de un sistema de información eficiente, la existencia de leyes laxas e imprecisas que admiten interpretaciones diferentes de la oficina jurídica y de las altas cortes, y por altos niveles de corrupción. Una DiAN eficiente en el control fiscal requiere superar esas carencias.

\section{Autonomía y planeación}

- La DiAn es hoy una unidad administrativa especial, adscrita y dependiente del Ministerio de Hacienda. Algo opuesto a la tendencia global a la independencia de estas entidades, que facilita el desarrollo de sus funciones y mejora los resultados del recaudo. En América Latina 11 de las 17 existentes son autónomas y en la OCDE 14 de las 20 existentes. La DIAN debería convertirse en una organización autónoma, para lo cual es necesario modificar la legislación respectiva.

- La administración de impuestos no puede improvisarse haciendo cambios sucesivos en la legislación, como ha sucedido: en quince años, las normas se han modificado diecisiete veces, y en ocho ocasiones se han llamado reformas tributarias, introduciendo 
soluciones coyunturales y cortoplacistas. La planeación se debe concebir a largo plazo.

\section{Recursos humanos}

- La DiAn es la entidad fiscal latinoamericana con menor número de empleados con respecto al de habitantes. Además, dedica el menor número de funcionarios a la fiscalización. Esta deficiencia facilita la evasión y la elusión, y se debe superar mediante un adecuado proceso de contratación de personal y de capacitación. El número de personas encargadas para hacer seguimiento a las empresas y personas naturales debería aumentar en forma razonable.

- No hay un verdadero plan de carrera en la DiAn, aunque esté escrito. Esta es una de sus principales debilidades y no favorece el clima organizacional. La posibilidad de hacer una carrera administrativa en cargos públicos siempre es un incentivo a la creatividad, el esfuerzo y la superación. Es necesario que la institución reglamente los ascensos.

\section{Tecnología y sistemas de información}

- La DIAN cuenta con dos grandes sistemas de información: el sYGA (siglo XXI) y el muisca. El primero sobre aduanas y el segundo sobre impuestos internos, pero no existe conexión entre ellos. En general, el Estado carece de un sistema de información nacional. El desacople entre los sistemas de las diversas entidades impide que la información fluya, dificulta su trabajo y hace posible la duplicidad de procedimientos, lo que genera ineficiencias para la entidad y para los contribuyentes. Es imperioso crear un sistema único de información tributaria y de contribuyentes.

- Una herramienta que ha contribuido a mejorar el control y a reducir la evasión y la elusión en el mundo es la factura electrónica. En Colombia este proceso ha sido muy lento y muy laxo. Según datos de la Cepal para 2015, Chile tenía un cubrimiento cercano al $80 \%$ y Perú del 18\%. En Colombia no hay cifras fiables, pero se considera inferior al 3\%, y es preciso superar el rezago.

\section{Control y fiscalización}

- En Colombia la fiscalización no es por sectores económicos ni por tipo de empresas, como en los países que ejercen un control adecuado. Hay una tímida sectorización de grandes contribuyentes en grupos amplios: servicios y operaciones financieras, sector 
manufacturero y químicos, hidrocarburos y minería, y comercio. Un auditor que un día va a una empresa de seguros al otro día puede ir a una empresa de lácteos. La sectorización debe ser menos amplia, por tipos de negocio y empresas específicas. E1 conocimiento específico facilita la auditoría y permite identificar en forma rápida y precisa las irregularidades.

\section{Penalización}

- Colombia es uno de los países más permisivos con el fraude fiscal. Los resultados de la fiscalización a veces son inocuos, pues ciertos contribuyentes que perciben que se ha detectado alguna irregularidad que han cometido, tienen tiempo suficiente para declararse insolventes y no pagar impuestos ni sanciones. La impunidad eleva la propensión a incurrir en fraude fiscal, así como sucede con el delito, con gran probabilidad de que no se sancione. Además, en el país no se encarcela a los defraudadores fiscales. Es conveniente que el país establezca pena de cárcel para delitos fiscales graves, como los castigan Chile, Argentina, México y Estados Unidos.

\section{Cooperación interinstitucional}

- E1 trabajo interinstitucional en el área tributaria es escaso: cada entidad trabaja por su parte a pesar de compartir objetivos e intereses similares. La DIAN, la policía fiscal y aduanera ( $\mathrm{POL}^{-}$ FA), las secretarías de hacienda municipal y departamental, el ICBF, el SENA y otras emplean información distinta, y carecen de coordinación. Incluso en la DIAN, la conexión entre el nivel que genera los programas y la base auditora es deficiente, y se elaboran programas poco realistas e ineficientes, lo que no favorece el recaudo ni reduce los espacios de fraude fiscal. Esta situación se debe afrontar siguiendo ejemplos como el de Alemania, donde las entidades nacionales y locales ejercen conjuntamente el control fiscal.

- Es necesario coordinar la organización tributaria nacional y las locales para cruzar datos de patrimonio, propiedades e ingresos, ejercer un mayor control y reducir la evasión a nivel nacional y local. Se requiere una estructura organizacional e institucional que armonice las instancias nacional, departamental y municipal, e incorpore en conjunto las bases de los impuestos de industria y comercio, predial, renta y patrimonio. 


\section{Cooperación internacional}

- Colombia debe tomar medidas estrictas para evitar la evasión de impuestos ocultando riquezas e ingresos en los países con los cuales no intercambia información y en paraísos fiscales. Según la DIAN, en 2012 solo se controlaba el 35\% de las operaciones internacionales. Como ejemplo, el gobierno no ha logrado un verdadero intercambio de información con Panamá, donde los colombianos tienen las mayores inversiones, sin un control de sus utilidades y su patrimonio. De modo que para disminuir la evasión tributaria es necesario ampliar los convenios de intercambio de información.

\section{Educación y cultura fiscal}

- La educación tributaria es casi inexistente. En una investigación realizada en colegios de Bogotá se concluyó que los escolares no sabían para qué sirven los impuestos, qué pasaba si los ciudadanos no pagan impuestos, si se sancionaba a quienes no los pagan, y que no entendían la gravedad de no pagarlos. El Estado, a través de la DiAn y del Ministerio de Educación, debe promover desde los primeros años escolares la educación y la cultura fiscal para que haya conciencia social de la importancia del pago de impuestos.

\section{Simplificación tributaria}

- La dian tiene poca capacidad para controlar la evasión y la elusión. En parte por la gran informalidad que hay en el país: una buena proporción de medianos y pequeños empresarios que obtienen incluso altas rentabilidades opera informalmente para evadir impuestos, y así reduce los ingresos fiscales. La simplificación tributaria debe ayudar a reducir esa informalidad. Para ello, en forma paralela a la emisión de facturas de las empresas grandes y medianas, se debe establecer la emisión de recibos para las pequeñas y muy pequeñas, como en Chile y Perú. En general, las empresas deberían rendir cuentas y liquidar impuestos ante la DIAN cada dos meses.

- Se debe simplificar el calendario tributario. La liquidación final y los pagos de los saldos adeudados del impuesto a las utilidades de las empresas, del impuesto de renta de personas naturales y del IVA deberían llevarse a cabo en el primer trimestre del año calendario. 


\section{SOBRE LOS EFECTOS ECONÓMICOS Y FISCALES DE LA REFORMATRIBUTARIA}

- No es sensato hacer una reforma tributaria sin prever sus efectos. Es necesario estimar su impacto sobre las variables económicas fundamentales: el crecimiento de la economía, los precios, la distribución del ingreso y, por supuesto, el recaudo. Hay al menos una razón clara: no tiene sentido una reforma que aumente el ingreso fiscal pero que genere recesión e inflación.

- Hay varias opciones para medir los efectos. A veces se emplean modelos económicos de equilibrio parcial centrados en el sistema fiscal, considerando ceteris paribus las demás variables económicas. Muchas veces ni siquiera se cuenta con ese tipo de modelos y se hacen estimaciones contables.

- Una manera adecuada de hacer esas estimaciones es emplear un modelo dinámico de equilibrio general computable (MEGC) que capte los efectos cruzados y la retroalimentación entre las diferentes variables económicas a lo largo del tiempo. El modelo que empleamos para estimar los efectos de nuestra propuesta fue construido específicamente para la economía colombiana, con un horizonte de proyección que llega a $2020^{2}$.

- Las proyecciones plenas del modelo empiezan a partir de 2016. Para años anteriores (2010-2015), de los que se dispone de información estadística parcial de muchas de las variables incluidas, y más completa para los años más antiguos, las estimaciones sirven para validar y calibrar el modelo. Las proyecciones de la reforma tributaria propuesta empiezan en 2017.

- Para la simulación y sus efectos sobre la economía hasta 2020, se consideraron dos escenarios que se compararon con un escenario base, la situación sin reforma. El primer escenario corresponde a nuestras propuestas, que sintetizamos en este escrito. El segundo escenario corresponde a la reforma tributaria que aprobó el Congreso en diciembre de 2016.

- Nuestra propuesta busca la equidad y la eficiencia en la tributación, sin descuidar el recaudo. También pretende minimizar la reducción de la tasa de crecimiento y los aumentos en los precios.

\footnotetext{
2 El megc fue elaborado por César Ferrari, con la asistencia de John Meisterl y Lina Quevedo, para la Comisión de Regulación de Comunicaciones de Colombia, que lo hizo de dominio público. La tabla de transacciones intersectoriales para Colombia en 2010, de la que parten las proyecciones del MEGC, fue construida por Jorge Centanaro. Ver Ferrari, C. "Modelo de equilibrio general computable (MEGC) dinámico para la economía colombiana con énfasis en el sector de tecnologías de la información y las comunicaciones (TIC)", diciembre de 2014, [www.crcom.gov.co/es/pagina/modelo-de-equilibrio-general-computable-megc].
} 
Sus características principales, en los términos antes señalados, incluyen:

- Mantener las tasas del IVA en su nivel actual, salvo para bebidas azucaradas y tabaco, que aumentarían, y reducir a cero la tasa de telefonía fija y celular así como de Internet fijo y móvil.

- Reducir el impuesto a las utilidades de las empresas y eliminar el CREE.

- Eliminar progresivamente el gravamen a los movimientos financieros.

- Establecer el impuesto a los dividendos.

- Aplicar el impuesto al patrimonio solo a personas naturales.

- Cobrar un impuesto a las remesas.

- Los aspectos principales de la reforma tributaria aprobada incluyen:

- Aumenta el iva de una tasa general del 16\% al 19\%, exceptuando la canasta básica que seguiría en $0 \%$, con un aumento mayor de la tasa al tabaco.

- Reduce la tasa nominal del impuesto a las utilidades de las empresas y elimina el CREE.

- Mantiene el gravamen a las transacciones financieras en una tasa del 4 por mil.

- Restablece el impuesto a los dividendos.

- Elimina el impuesto a la riqueza de personas naturales y jurídicas.

- En términos generales, las simulaciones realizadas con el MEGC muestran que un mayor incremento de las tasas de impuestos aumenta el ingreso fiscal pero provoca una desaceleración de la economía y una elevación de precios, como sugiere la teoría más aceptada. De modo que una reforma tributaria adecuada no solo debería lograr un mayor recaudo sino que debería tener efectos positivos sobre las variables fundamentales: crecimiento, estabilidad de precios y distribución del ingreso. Ello implica aumentar algunas tasas y, seguramente, reducir otras.

- Los cuadros 4 y 5 presentan los principales resultados del ejercicio. El cuadro 4 muestra los resultados de la propuesta de reforma y los compara con los del escenario base. El cuadro 5 presenta las proyecciones correspondientes a la reforma aprobada en diciembre de 2016, también comparadas con las del escenario base.

- Los resultados que arroja el MEGC para nuestra propuesta no son sorprendentes dadas sus características, en particular no aumentar 
el iva, salvo en los casos indicados. Comparados con los que se obtendrían en el escenario base -sin reforma-, los ingresos tributarios se verían casi inalterados en el primer año de la reforma (reducción de 5 mil millones de pesos). Sin embargo, en los años siguientes serían muy superiores (9,9 billones de pesos en 2018, 18,0 billones en 2019 y 20,8 billones en 2020). De modo que el recaudo promedio anual en los cuatro años de proyección de la propuesta sería mucho mayor que en el escenario base $(12,2 \mathrm{bi}-$ llones de pesos más).

- La consecuencia sería una reducción del déficit fiscal como proporción del рів. Frente al escenario base, el déficit tendría un pequeño aumento en 2017 (0,02 puntos porcentuales), pero disminuiría en los años siguientes $(0,81$ puntos menos en $2018,1,61$ puntos en 2019 y 1,81 puntos en 2020).

- Estos resultados obedecen en gran medida a los nuevos impuestos a los dividendos y al patrimonio de las personas naturales, que a partir del segundo año más que compensarían la eliminación del CREE, la reducción de la tasa del impuesto a la renta de las empresas y la reducción progresiva del impuesto a los movimientos financieros.

- Por su parte, según el MEgc, la reforma tributaria aprobada produciría un mayor recaudo frente al que se obtendría en el escenario base (9,3 billones de pesos más en 2017; 7,7 billones en 2018; 8,3 billones en 2019, y 12,2 billones en 2020). Pero el aumento promedio anual durante los años de la proyección con respecto al escenario base sería inferior al de nuestra propuesta $(9,4$ billones frente a 12,2 billones, respectivamente).

- La reforma aprobada reduciría el déficit fiscal durante todos los años de la proyección (1,02 puntos porcentuales en 2017; 0,39 en 2018; 0,40 en 2019, y 0,74 en 2020). Una reducción ocasionada por la principal medida aprobada: el aumento de la tarifa general de iva del 16 al 19\% por una única vez en 2017.

- Por su parte, nuestra propuesta provocaría un aumento de la tasa de inflación durante el primer año con respecto a la proyección del escenario base (1,3 puntos porcentuales más en 2017). En los años siguientes la inflación sería menor que en el escenario base (0,92 puntos porcentuales menos en 2018; 0,70 en 2019, y 0,82 en 2020).

- También provocaría una baja de la tasa de crecimiento del PIB frente al escenario base en el primer año $(1,4$ puntos porcentuales menos), aunque sería mayor en los años siguientes (1,2 puntos más 
en 2018; 0,10 en 2019, y 0,56 en 2020). A su vez, el Gini después de impuestos disminuiría durante todos los años de proyección en $0,5,1,9,2,0$ y 2,3 puntos porcentuales, respectivamente.

- La razón de esos resultados es evidente: el aumento de los precios induciría una contracción de la demanda que luego sería compensada por la mayor inversión de las empresas debido a la reducción del impuesto a la renta y la supresión del impuesto al patrimonio, que tendrían además efectos redistributivos sobre el ingreso.

- En cambio, la reforma tributaria aprobada induciría un aumento de la inflación mucho mayor en 2017 (2,6 puntos porcentuales más) con respecto al escenario base que el que provocaría nuestra propuesta. Sin embargo, las tasas de los años siguientes serían ligeramente menores que las del escenario base $(0,06$ puntos en 2018; 0,42 en 2019, y 0,58 en 2020).

- A su vez, frente al escenario base, la reforma aprobada produciría una fuerte caída de la tasa de crecimiento del piв en $2017(2,3$ puntos porcentuales menos), una reducción menor en $2018(0,80$ puntos) y leves aumentos en los dos años siguientes ( 0,16 puntos en 2019 y 0,34 en 2020). Además, la reforma empeoraría la distribución del ingreso: el Gini después de impuestos aumentaría todos los años de la proyección, en 1,4,2,0,1,9 y 1,4 puntos porcentuales, respectivamente.

- La razón de los resultados de la reforma aprobada es clara: el aumento del iva a todos los bienes y servicios (excepto a los de la canasta familiar) eleva su precio, lo que contrae la demanda y, por tanto, reduce el nivel de actividad económica y deteriora la distribución del ingreso. Ese efecto no es compensado por la reducción del impuesto de renta a las empresas, que no provoca un alza suficiente de la inversión y lleva a un crecimiento inferior.

- Cabe hacer una aclaración final: el MEGC no es una bola de cristal ni un medio para adivinar el futuro. Tampoco es la realidad. Es una representación simplificada que reproduce, con cierta aproximación, su comportamiento siguiendo las reglas de la teoría económica. Además, no incorpora los cambios de comportamiento de los agentes económicos en reacción a los cambios de política económica, en este caso a los nuevos impuestos. De tal modo, cualquier resultado del modelo es una aproximación que depende de esas premisas teóricas y, en gran medida, de cuán acertados sean sus supuestos exógenos (de comportamiento, precios internacionales e instrumentos de política). 


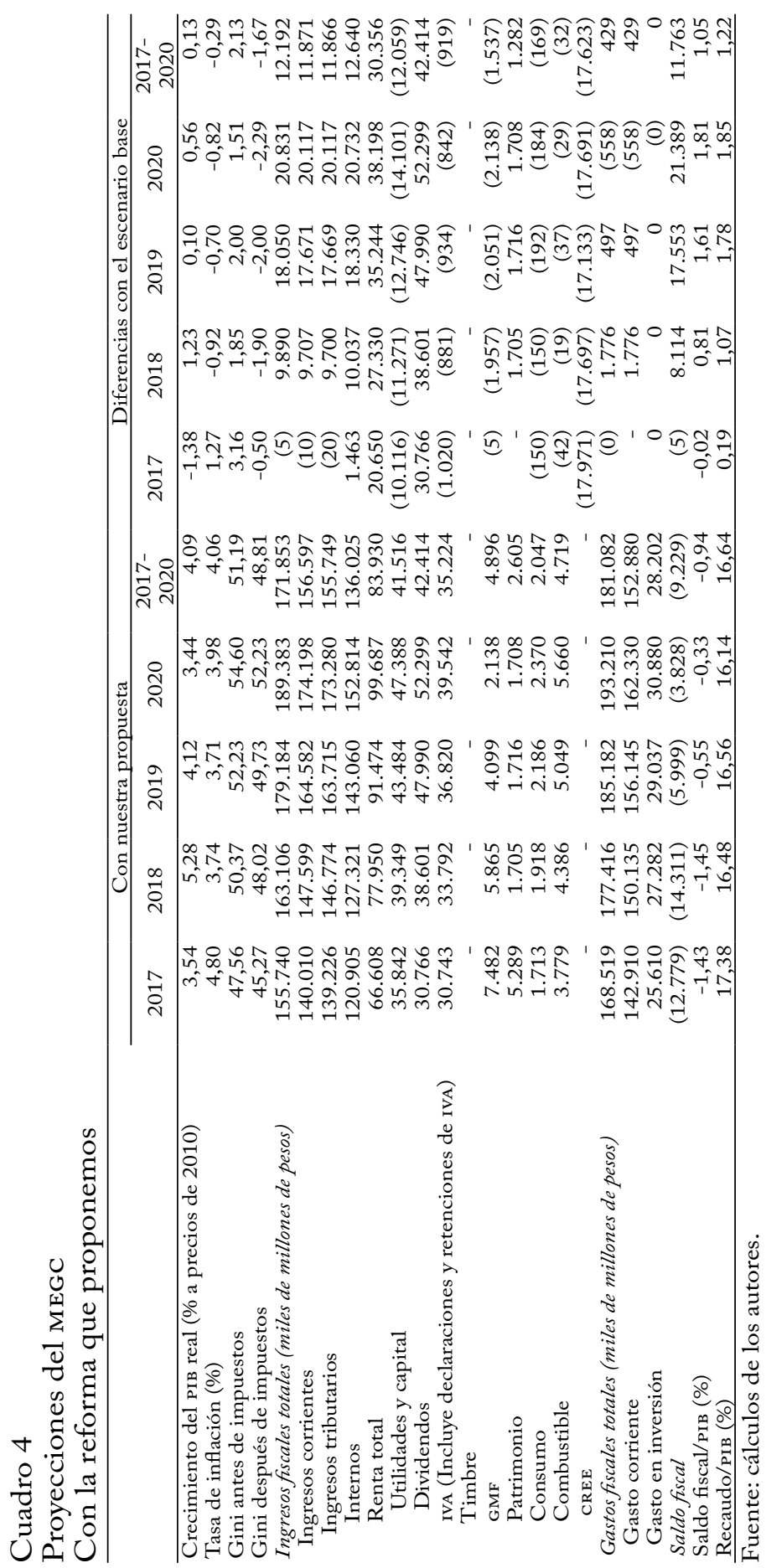

Revista de Economía Institucional, vol. i9, n.o 36, Primer semestre/2oi7, pp. I49-i74 


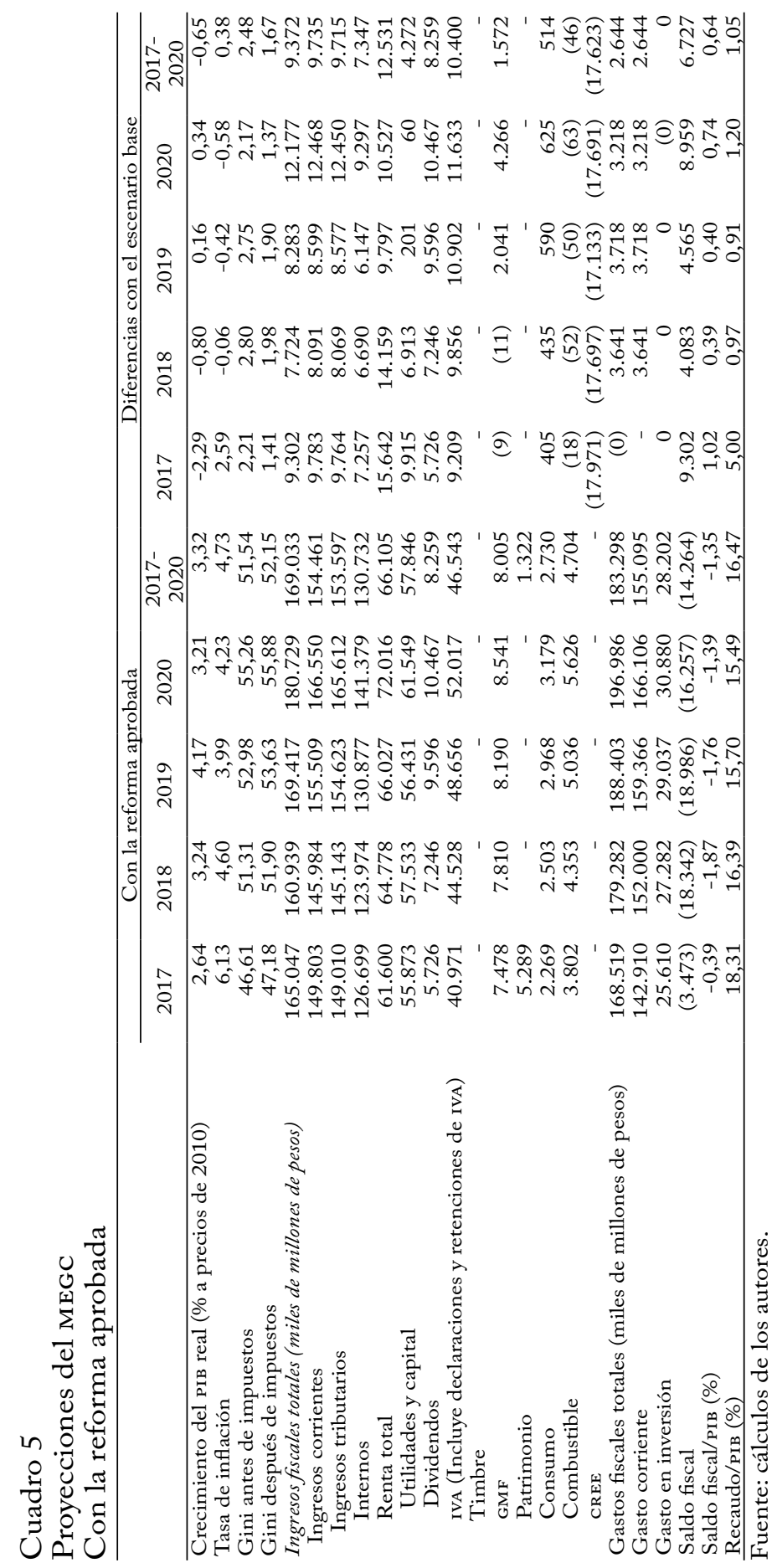

Revista de Economía Institucional, vol. i9, N.o 36, primer semestre/2oi7, pp. I49-i74 